\title{
Prognostic Significance of Survivin Expression and Combined Analysis with Cancer Stem Cell and Epithelial-Mesenchymal Transition-related Markers in Patients with Rectal Cancer Undergoing Preoperative Chemoradiotherapy
}

\author{
JIYOUNG KIM ${ }^{1 *}$, SOOMIN AHN ${ }^{2 *}, \mathrm{KYUBO} \mathrm{KIM}^{1}$, MIN SUN CHO ${ }^{3}$, \\ KWANG HO KIM ${ }^{4}$, RYUNG-AH LEE ${ }^{4}$ and EUN MI NAM ${ }^{5}$ \\ ${ }^{1}$ Department of Radiation Oncology, Ewha Womans University College of Medicine, Seoul, Republic of Korea; \\ ${ }^{2}$ Department of Pathology, Seoul National University Bundang Hospital, Seongnam, Republic of Korea; \\ ${ }^{3}$ Department of Pathology, Ewha Womans University College of Medicine, Seoul, Republic of Korea; \\ ${ }^{4}$ Department of Surgery, Ewha Womans University College of Medicine, Seoul, Republic of Korea; \\ ${ }^{5}$ Department of Internal Medicine, Ewha Womans University College of Medicine, Seoul, Republic of Korea
}

\begin{abstract}
Aim: To identify the candidate marker predicting treatment response and survival outcome in rectal cancer patients who received preoperative chemoradiotherapy (CRT). Patients and Methods: Between 2000 and 2015, 159 patients with histologically-confirmed rectal adenocarcinoma underwent preoperative CRT followed by surgery. Among them, 70 patients were enrolled and the expression of survivin, cancer stem cell markers (CD44 and CD133) and epithelial-mesenchymal transition markers (E-cadherin and TWIST1) in pretreatment biopsy specimens were evaluated by immunohistochemistry. Associations between the expression of markers and clinical outcomes were evaluated. Results: The median follow-up period of all patients was 71 (range=15-203) months. Five-year overall (OS), disease-free (DFS), locoregional recurrence-free (LRRFS) and distant metastasis-free (DMFS) survival were $80.5 \%, 60.2 \% 90.1 \%$
\end{abstract}

*These Authors contributed equally to this study.

Correspondence to: Kyubo Kim, MD, Ph.D., Department of Radiation Oncology, Ewha Womans University College of Medicine, 1071, Anyangcheon-ro, Yangcheon-gu, Seoul, Republic of Korea. Tel: +82 226505334, Fax: +82 226540363, e-mail: kyubokim.ro@gmail.com and Min Sun Cho, MD, Ph.D., Department of Pathology, Ewha Womans University College of Medicine, 1071, Anyangcheon-ro, Yangcheon-gu, Seoul, Republic of Korea. Tel: +82 226505044, Fax: +82 226542016, e-mail: mcho1124@ewha.ac.kr

Key Words: Rectal cancer, preoperative chemoradiotherapy, survivin, cancer stem cell, epithelial-mesenchymal transition, immunohistochemistry. and $76.5 \%$, respectively. A significant association between survivin overexpression and worse treatment outcome was shown on univariate analyses for OS, DFS and DMFS ( $p=0.022,0.002$, and 0.005 , respectively). On multivariate analysis, survivin overexpression was an adverse prognosticator for DFS and DMFS ( $p=0.007$ and 0.015, respectively), with a borderline significant trend towards a shorter $O S(p=0.069)$. Four other single biomarkers were not associated with survival outcomes. However, overexpression of both survivin and CD44 was significantly associated with worse OS on multivariate analysis ( $p=0.003)$. Conclusion: Survivin combined with CD44 might be a candidate biomarker for the prediction of recurrence and survival in patients who received preoperative CRT for rectal cancer. Further research with a larger population is needed to validate these results.

The traditional management of locally advanced rectal cancer was surgery followed by postoperative chemoradiotherapy (CRT) (1). In comparison with postoperative CRT, however, preoperative CRT has the advantage of lower toxicity, a higher sphincter preservation rate, and a lower locoregional recurrence rate. These benefits were demonstrated in a randomized trial, therefore multimodality treatment including preoperative CRT and surgical resection with or without adjuvant chemotherapy has been established as a standard of care for locally advanced rectal cancer (2-4). However, this treatment strategy is not equally effective for all patients. In spite of multimodality therapy, $30-40 \%$ of patients experience relapse $(2,3)$. Treatment failure and heterogeneity of prognosis is probably due to biological properties of the tumor (5). Hence, a number of studies have evaluated 
potential biomarkers based on the hallmarks of cancer, and several proteins functioning in apoptosis, proliferation, angiogenesis, DNA repair, hypoxia, metabolism, and pluripotency have been suggested as possible prognostic biomarkers for rectal cancer in patients who received preoperative CRT (5-8).

Among these biomarkers, survivin has been found to be one of the most intriguing. Survivin, a member of the inhibitor of apoptosis protein family, plays an important role in suppressing caspase-mediated apoptosis (9). Recently, a meta-analysis revealed that survivin overexpression was significantly associated with poorer overall survival (OS) and a metastatic capacity in patients with colorectal cancer $(10$, 11). In addition, much evidence indicates that expression of cancer stem cell (CSC)/epithelial-mesenchymal transition (EMT) markers were correlated with aggressive behavior of carcinoma and a ---poor prognosis $(12,13)$. However, the relationship between CSC/EMT marker expression and prognosis remains controversial in rectal cancer. In recent years, co-expression of promising markers has demonstrated an enhanced role in predicting prognosis $(14,15)$. However, no data are presently available concerning the co-expression of survivin and CSC/EMT markers in rectal cancer. We assumed that combined use of these markers may better predict treatment response and prognosis.

The purpose of this study was to investigate survivin, CD44, CD133, E-cadherin and TWIST1 expression in patients who underwent preoperative CRT for rectal cancer, and identify their relations to treatment response and prognosis. In addition, the prognostic significance of combined expression of these markers was evaluated.

\section{Patients and Methods}

Patients. Between 2000 and 2015, 159 patients with locally advanced rectal cancer underwent preoperative CRT with 50.4 Gy of pelvic irradiation followed by surgery. Patients whose biopsies were performed at outside institutions, who refused operation or with follow-up loss were excluded. As a result, 70 pretreatment tumor biopsy specimens were available. Medical records were reviewed to obtain clinicopathological parameters such as sex, age, comorbidity, date of first diagnosis, hematological profile, carcinoembryonic antigen (CEA), preoperative CRT data, surgery type, tumor size, tumor differentiation, presence of lymphovascular invasion, presence of perineural invasion, circumferential resection margin status and TNM stage according to the American Joint Committee on Cancer seventh edition (16). Information on locoregional recurrence, distant metastasis, and survival were also retrieved. The histological features from all biopsies and resected specimens were reassessed by a specialized pathologist blinded to clinical information. This study was approved by the Institutional Review Board (approval number; 2016-03-058).

Immunohistochemical staining. Immunohistochemical staining for survivin (dilution 1:100; Santa Cruz Biotechnology, Santa Cruz, CA, USA), CD44 (dilution 1:100; Leica Biosystems, Novocastra,
Newcastle-upon-Tyne, UK), CD133 (dilution 1:100; Novus Biologicals, Littleton, Colorado, USA), E-cadherin (dilution 1:100; Leica Biosystems, Novocastra) and TWIST1 (dilution 1:200; Abcam, Cambridge, MA, USA) was carried out using a Bond-max autoimmunostaining system (Leica Biosystem, Melbourne, Australia) with Bond ${ }^{\mathrm{TM}}$ Polymer refine detection, DS9800 kit (Leica Biosystems, Melbourne, Australia). Four-micrometer-thick sections from formalin-fixed, paraffin embedded pretreatment tumor biopsy specimens were transferred to adhesive slides and dried at $62^{\circ} \mathrm{C}$ for $30 \mathrm{~min}$. The slides were then deparaffinized and endogenous peroxidase was quenched by incubating the tissue with $3 \%$ hydrogen peroxide for $10 \mathrm{~min}$. Antigen retrieval was performed using Bond Epitope Retrieval solution for $20 \mathrm{~min}$ at $9^{\circ} \mathrm{C}$. Sections were incubated with primary antibodies for $15 \mathrm{~min}$, the post-primary antibody for $10 \mathrm{~min}$, and polymer for $30 \mathrm{~min}$, followed by 3,3'diaminobenzidine and counterstaining with hematoxylin.

Immunohistochemistry analysis. Survivin, CD133, and TWIST1 were expressed in nuclei and both the intensity (0, none; 1 , weak; 2 , moderate; 3 , strong staining) and proportion of positively stained cells $(0,<5 \% ; 1,5-24 \% ; 2,25-49 \% ; 3,50-74 \% ; 4, \geq 75 \%)$ were assessed. For survivin, the staining intensity and percentage of positively stained tumor cells were multiplied to produce a weighted score for each case and dichotomized, according to Sinicrope et al. (17). Tumors with a weighted score of 0 to 4 were considered to have low expression, while those with scores of 5 to 12 were considered to have high expression. For evaluation of CD133 and TWIST1 expression, the sum of the intensity score and proportion score were calculated, according to Zhang et al. (18) and Zhu et al. (19). Scores of 0 to 3 were defined as low expression, while scores of 4 to 7 were defined as high expression. E-Cadherin and CD44 exhibited membranous staining. ECadherin was assessed by intensity (0, normal; 1 , mild loss; 2, moderate loss; 3 , complete loss). It was subsequently categorized into intact (scores of 0 and 1 ) or loss (scores of 2 and 3 ) of expression. CD44 was also assessed by intensity ( 0 , negative; 1 , weak; 2 , moderate; 3 , strong) and was categorized into low expression (scores of 0 and 1 ) or high expression (scores of 2 and 3 ).

Assessment of treatment response. Response to preoperative CRT was evaluated in the resected specimens. According to grading system described by Dworak, tumor regression grade (TRG) was evaluated as following: TRG0, No regression (no evidence of change); TRG1, minimal regression (dominant tumor mass with obvious change); TRG2, moderate regression (dominant change with residual tumor); TRG3, near total regression (microscopic residual tumor in fibrotic tissue); TRG4, total regression (no residual tumor cells, only fibrotic mass). Patients with TRG0 and 1 were grouped as non-responders, while those with TRG2, -3 and -4 were grouped as responders. Pathological complete remission was defined as no detection of any residual cancer cells in the resection specimen. Tumor down-staging was characterized by evidence of reduction in $\mathrm{T}$ or $\mathrm{N}$ categories.

Statistical analysis. Pearson chi-square tests or Fisher's exact tests were used to identify statistically significant differences between categorical variables. Correlations between expressions of molecular markers were analyzed using Pearson correlation coefficients. Kaplan-Meier analysis was used to estimate the rates of overall (OS), disease-free (DFS), locoregional recurrence-free (LRFS) and distant metastasis-free (DMFS) survival. A log-rank test was 
performed to test for differences in the survival curves. All time-toevent endpoints were calculated from the date of biopsy and recurrences were determined by imaging. The Cox proportional hazard regression model was used for univariate and multivariate analyses. Prognostic factors with a $p$-value of less than 0.2 on the univariate analysis were included in the multivariate model using forward selection. Statistical tests were performed with SPSS software version 18.0.0 (SPSS, Chicago, IL, USA), and $p$-values of less than 0.05 were considered significant. Cut-off values for CSC/EMT markers have varied in previous studies and there were no commonly used criteria. Hence, the cut-off values in this study were determined by a maximally selected rank statistics using the $\mathrm{R}$ statistical software package version 3.0.2 ( $\mathrm{R}$ Foundation for Statistical Computing, Vienna, Austria).

\section{Results}

Patient characteristics and immunohistochemical expression of markers. The patient characteristics are summarized in Table I. The median age was 57 (range=26-82) years. Fortyfour patients $(62.9 \%)$ were males and 26 patients were females $(37.1 \%)$. The majority of patients had clinical stage T3/T4 (67 patients, 95.7\%) and clinically positive lymph nodes were noted in 52 patients $(74.3 \%)$.

Figure 1 shows representative cases of immunohistochemical staining. For survivin, 22 patients (31.4\%) were included in the high-survivin expression group. Forty-four out of 70 patients $(62.9 \%)$ were categorized as having high CD44 expression, and 35 patients $(50.0 \%)$ were categorized as high CD133 expression. Fifty-eight patients (82.9\%) were categorized with E-cadherin loss, and 40 patients $(57.1 \%)$ were categorized into the high TWIST1 expression group. There was no significant correlation between the expression of markers and clinicopathological factors.

Correlation between expression of markers and treatment response. Pathological complete remission was observed in 10 patients $(14.3 \%)$, TRG3 in $21(30.0 \%)$, TRG2 in 22 (31.4\%), TRG1 in $16(22.9 \%)$, and no regression in one (1.4\%). T down-staging was observed in 38 patients $(54.3 \%)$ and $\mathrm{N}$ down-staging was observed in 31 (44.3\%). Among five markers, only survivin expression was negatively associated with TRG $(p=0.031)$. Expression of molecular markers was not related to down-staging (Table II).

Correlation between expression of markers and survival. The median follow-up period of patients overall was 71 (range $=15$ 203) months. During the follow-up period, cancer relapse occurred in 20 patients $(28.6 \%)$ : isolated distant metastasis occurred in 14 patients $(20.0 \%)$, isolated locoregional recurrence in five $(7.1 \%)$ and one patient $(1.4 \%)$ experienced both local and distant progression. Five-year OS, DFS, LRFS and DMFS were $80.5 \%, 60.2 \% 90.1 \%$ and $76.5 \%$, respectively. Univariate analysis indicated that high expression of survivin $(p=0.022), \mathrm{CEA}>5 \mathrm{ng} / \mathrm{ml}(p=0.031), \mathrm{ypT} 3 / 4$ disease $(p=0.024)$,
Table I. Patient characteristics.

\begin{tabular}{|c|c|c|}
\hline Variable & No. & $\%$ \\
\hline \multicolumn{3}{|l|}{ Gender } \\
\hline Male & 44 & 62.9 \\
\hline Female & 26 & 37.1 \\
\hline \multicolumn{3}{|l|}{ Age } \\
\hline$\leq 50$ Years & 14 & 20 \\
\hline$>50$ Years & 56 & 80 \\
\hline \multicolumn{3}{|l|}{ CEA } \\
\hline$\leq 5 \mathrm{ng} / \mathrm{ml}$ & 39 & 55.7 \\
\hline$>5 \mathrm{ng} / \mathrm{ml}$ & 31 & 44.3 \\
\hline \multicolumn{3}{|l|}{ Clinical $\mathrm{T}$ category } \\
\hline $\mathrm{T} 1-2$ & 3 & 4.3 \\
\hline T3-4 & 67 & 95.7 \\
\hline \multicolumn{3}{|l|}{ Clinical N category } \\
\hline Negative & 18 & 25.7 \\
\hline Positive & 52 & 74.3 \\
\hline \multicolumn{3}{|l|}{ Chemotherapy regimen } \\
\hline FL & 61 & 87.1 \\
\hline Capecitabine & 6 & 8.6 \\
\hline FOLFOX & 3 & 4.3 \\
\hline \multicolumn{3}{|l|}{ Surgery } \\
\hline Transanal excision & 5 & 7.1 \\
\hline Low anterior resection & 47 & 67.1 \\
\hline Abdominoperineal resection & 18 & 25.7 \\
\hline \multicolumn{3}{|l|}{ Differentiation $^{\mathrm{a}}$} \\
\hline WD, MD & 53 & 75.7 \\
\hline PD & 7 & 10.0 \\
\hline \multicolumn{3}{|l|}{ Lymphatic invasion $^{\mathrm{a}}$} \\
\hline Absent & 45 & 64.3 \\
\hline Present & 15 & 21.4 \\
\hline \multicolumn{3}{|l|}{ Perineural invasion ${ }^{\mathrm{a}}$} \\
\hline Absent & 48 & 68.6 \\
\hline Present & 12 & 17.1 \\
\hline \multicolumn{3}{|l|}{ Circumferential resection margin ${ }^{a}$} \\
\hline Negative & 58 & 82.9 \\
\hline Positive & 2 & 2.9 \\
\hline \multicolumn{3}{|l|}{ Pathological $\mathrm{T}$ category } \\
\hline T0 & 10 & 14.3 \\
\hline $\mathrm{T} 1$ & 6 & 8.6 \\
\hline $\mathrm{T} 2$ & 20 & 28.6 \\
\hline $\mathrm{T} 3$ & 33 & 47.1 \\
\hline $\mathrm{T} 4$ & 1 & 1.4 \\
\hline \multicolumn{3}{|l|}{ Pathological N category $\mathrm{b}$} \\
\hline No & 43 & 61.4 \\
\hline N1 & 14 & 20.0 \\
\hline N2 & 6 & 8.6 \\
\hline \multicolumn{3}{|l|}{ Adjuvant chemotherapy } \\
\hline No & 12 & 17.1 \\
\hline Yes & 58 & 82.9 \\
\hline
\end{tabular}

CEA, Carcinoembryonic antigen; FL, 5-fluorouracil plus leucovorin; FOLFOX, 5-fluorouracil plus oxaliplatin; WD, welldifferentiated; MD, moderately differentiated; P/D, poorly differentiated. aEvaluations were performed in patients without pathological complete response. ${ }^{b} \mathrm{Lymph}$ node evaluation was performed in 63 patients $(90.0 \%)$.

poor differentiation $(p=0.029)$, TRG0-1 $(p=0.002)$, no downstaging $(p=0.037)$, and positivity of the circumferential resection margin $(p<0.001)$ were associated with a shorter OS. 

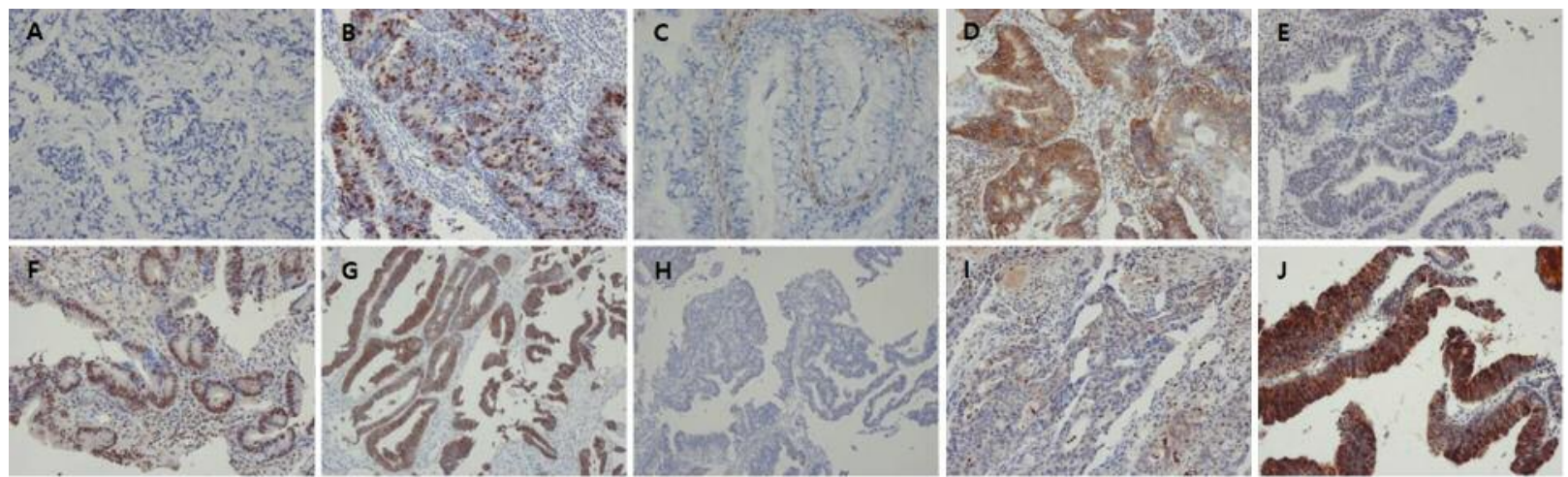

Figure 1. Representative immunohistochemical staining of markers. Survivin: Weak intensity (A), moderate intensity (B) $(\times 200)$. CD44: Negative $(C)$, strong intensity $(D)(\times 200)$. CD133: Weak intensity $(E)$, strong intensity $(F)(\times 200)$. E-Cadherin: Preserved expression $(G)(\times 100)$, complete loss of intensity $(H)(\times 100)$. TWIST1: Weak intensity $(I)$, strong intensity $(J)(\times 200)$.

Table II. Correlation between expression of markers and treatment response.

\begin{tabular}{|c|c|c|c|c|c|c|c|c|c|c|}
\hline \multirow[b]{2}{*}{ Marker } & \multirow[b]{2}{*}{ Expression } & \multicolumn{3}{|c|}{ TRG, n (\%) } & \multicolumn{3}{|c|}{ T Down-staging, n (\%) } & \multicolumn{3}{|c|}{ N Down-staging, $\mathrm{n}(\%) \mathrm{a}$} \\
\hline & & $0-1(n=17)$ & $2-4(n=53)$ & $p$-Value & No $(n=32)$ & Yes $(n=38)$ & $p$-Value & No $(n=32)$ & Yes $(n=31)$ & $p$-Value \\
\hline \multirow[t]{2}{*}{ Survivin } & Low & $8(47.1 \%)$ & $40(75.5 \%)$ & 0.031 & $20(62.5 \%)$ & $28(73.7 \%)$ & 0.228 & $23(71.9 \%)$ & $20(64.5 \%)$ & 0.361 \\
\hline & High & $9(52.9 \%)$ & $13(24.5 \%)$ & & $12(37.5 \%)$ & $10(26.3 \%)$ & & $9(28.1 \%)$ & $11(35.5 \%)$ & \\
\hline \multirow[t]{2}{*}{ CD44 } & Low & $6(35.3 \%)$ & $20(37.7 \%)$ & 0.548 & $16(46.9 \%)$ & $11(28.9 \%)$ & 0.097 & $13(40.6 \%)$ & $9(29.0 \%)$ & 0.242 \\
\hline & High & $11(64.7 \%)$ & $33(62.3 \%)$ & & $17(53.1 \%)$ & $27(71.1 \%)$ & & $19(59.4 \%)$ & $22(71.0 \%)$ & \\
\hline \multirow[t]{2}{*}{ CD133 } & Low & $7(41.2 \%)$ & $28(52.8 \%)$ & 0.289 & $13(40.6 \%)$ & $22(57.9 \%)$ & 0.115 & $17(53.1 \%)$ & $16(51.6 \%)$ & 0.552 \\
\hline & High & $10(58.8 \%)$ & $25(47.2 \%)$ & & $19(59.4 \%)$ & $16(42.1 \%)$ & & $15(46.9 \%)$ & $15(48.4 \%)$ & \\
\hline \multirow[t]{2}{*}{ E-Cadherin } & Mild loss & $3(17.6 \%)$ & $9(17.0 \%)$ & 0.604 & $7(21.9 \%)$ & $5(13.2 \%)$ & 0.259 & $9(28.1 \%)$ & $3(9.7 \%)$ & 0.060 \\
\hline & Complete loss & $14(82.4 \%)$ & $44(83.0 \%)$ & & $25(78.1 \%)$ & $33(86.8 \%)$ & & $23(71.9 \%)$ & $28(90.3 \%)$ & \\
\hline \multirow[t]{2}{*}{ TWIST1 } & Low & $5(29.4 \%)$ & $25(47.2 \%)$ & 0.157 & $11(34.4 \%)$ & $19(50.0 \%)$ & 0.141 & $14(43.8 \%)$ & $14(45.2 \%)$ & 0.556 \\
\hline & High & $12(70.6 \%)$ & $28(52.8 \%)$ & & $21(65.6 \%)$ & $19(50.0 \%)$ & & $18(56.3 \%)$ & $17(54.8 \%)$ & \\
\hline
\end{tabular}

TRG, Tumor regression grade; a Lymph node evaluation was performed in 63 patients $(90.0 \%)$. Bold values indicate significant difference.

Survivin overexpression ( $p=0.002)$, TRG0-1 $(p=0.038)$, and circumferential resection margin positivity $(p=0.002)$ were also associated with a shorter DFS. Survivin overexpression was the only factor associated with a shorter DMFS ( $p=0.005$; Table III) whereas it was not associated with LRFS $(p=0.258)$. Figure 2 shows the Kaplan-Meier curves for OS, DFS, LRFS, and DMFS according to survivin expression. On multivariate analysis, TRG0-1 $(p=0.026)$ and circumferential resection margin positivity $(p=0.002)$ were independent predictors for OS. High expression of survivin was an independent prognostic factor for DFS and DMFS ( $p=0.007$ and 0.015 , respectively) and had a borderline significant trend towards a shorter OS ( $p=0.069$; Table III). The four other single biomarkers were not associated with survival outcomes (Table IV).

Prognostic value of combined marker analysis. Although CD44, CD133, E-cadherin and TWIST1 expression status did not predict OS ( $p=0.279,0.569,0.346$, and 0.374 respectively), the combined analysis of CSC/EMT markers with survivin showed significant association with OS (Table IV). Most significant prognostic biomarker combinations included coexpression of both survivin and CD44. On univariate analysis, phenotype for positivity for survivin and CD44 was a significant predictor for OS and DFS ( $p=0.001$ and 0.010 , respectively; Figure 3 ). In addition, multivariate Cox proportional hazard model demonstrated that this phenotype remained an independent predictor for OS (hazard ratio $=6.45$; $95 \%$ confidence interval, 1.87 to $22.25, p=0.003$; Table V).

\section{Discussion}

In the present study, we examined the expression of survivin, CSC (CD44 and CD133) and EMT (E-cadherin and TWIST1) markers in pretreatment biopsy specimens in patients who received preoperative CRT for rectal cancer. Survivin overexpression was an independent prognostic 
Table III. Univariate and multivariate analyses for overall (OS), disease-free (DFS) and distant metastasis-free (DMFS) survival.

\begin{tabular}{|c|c|c|c|c|c|c|c|c|c|c|c|c|}
\hline \multirow[b]{3}{*}{ Variable } & \multicolumn{4}{|c|}{ OS } & \multicolumn{4}{|c|}{ DFS } & \multicolumn{4}{|c|}{ DMFS } \\
\hline & UVA & & MVA & & UVA & & MVA & & UVA & & MVA & \\
\hline & $p$-Value & HR & $95 \% \mathrm{CI} \quad l$ & $p$-Value & $p$-Value & HR & $95 \% \mathrm{CI}$ & $p$-Value & $p$-Value & HR & $95 \% \mathrm{CI}$ & $p$-Value \\
\hline \multicolumn{13}{|l|}{ Survivin } \\
\hline High vs. low & 0.022 & 2.96 & $0.92-9.52$ & 0.069 & 0.002 & 3.30 & $1.40-7.79$ & 0.007 & 0.005 & 3.74 & $1.30-10.79$ & 0.015 \\
\hline \multicolumn{13}{|l|}{ Gender } \\
\hline Male $v s$. female & 0.392 & & & & 0.566 & & & & 0.471 & & & \\
\hline \multicolumn{13}{|l|}{ Age } \\
\hline$>50 v s . \leq 50$ Years & 0.140 & 1.91 & $0.36-10.11$ & 0.447 & 0.449 & & & & 0.551 & & & \\
\hline \multicolumn{13}{|l|}{ CEA } \\
\hline$>5 \mathrm{ng} / \mathrm{ml} v s . \leq 5 \mathrm{ng} / \mathrm{ml}$ & 0.031 & 2.88 & $0.88-9.42$ & 0.080 & 0.339 & & & & 0.762 & & & \\
\hline \multicolumn{13}{|l|}{ Pathological T category } \\
\hline T3-4 vs. T0-2 & 0.024 & 0.53 & $0.11-2.63$ & 0.438 & 0.169 & 0.62 & $0.23-1.71$ & 0.356 & 0.251 & & & \\
\hline \multicolumn{13}{|l|}{ Pathological N category } \\
\hline N1-2 vs. N0 & 0.705 & & & & 0.972 & & & & 0.835 & & & \\
\hline \multicolumn{13}{|l|}{ Differentiation } \\
\hline $\mathrm{PD} v s . \mathrm{WD} / \mathrm{MD}$ & 0.029 & 2.66 & $0.62-11.38$ & 0.188 & 0.112 & 1.97 & $0.57-6.82$ & 0.283 & 0.645 & & & \\
\hline \multicolumn{13}{|l|}{ Lymphatic invasion } \\
\hline Present vs. absent & 0.960 & & & & 0.799 & & & & 0.656 & & & \\
\hline \multicolumn{13}{|l|}{ Perineural invasion } \\
\hline Present $v s$. absent & 0.101 & 0.47 & $0.09-2.39$ & 0.472 & 0.264 & & & & 0.580 & & & \\
\hline \multicolumn{13}{|l|}{$\begin{array}{l}\text { Circumferential } \\
\text { resection margin }\end{array}$} \\
\hline Positive $v s$. negative & $<0.001$ & 40.49 & $3.74-437.85$ & 50.002 & 0.002 & 3.20 & $0.44-23.21$ & 0.250 & 0.082 & 4.13 & $0.48-34.76$ & 0.192 \\
\hline \multicolumn{13}{|l|}{ TRG } \\
\hline $2-4$ vs. $0-1$ & 0.002 & 0.18 & $0.04-0.81$ & 0.026 & 0.038 & 0.47 & $0.15-1.40$ & 0.173 & 0.975 & & & \\
\hline \multicolumn{13}{|l|}{ T Down-staging } \\
\hline Yes $v s$. no & 0.037 & 0.69 & $0.17-2.85$ & 0.603 & 0.252 & & & & 0.440 & & & \\
\hline \multicolumn{13}{|l|}{ N Down-staging } \\
\hline Yes vs. no & 0.211 & & & & 0.849 & & & & 0.500 & & & \\
\hline \multicolumn{13}{|l|}{ Adjuvant chemotherapy } \\
\hline No $v s$. yes & 0.213 & 1.26 & $0.25-6.28$ & 0.778 & 0.206 & & & & 0.221 & & & \\
\hline
\end{tabular}

UVA, Univariate analysis; MVA, multivariate analysis; HR, hazard ratio; CI, confidence interval; CEA, carcinoembryonic antigen; PD, poorly differentiated; WD, welldifferentiated; MD, moderately differentiated; TRG, tumor regression grade. Bold values indicate significant difference.

factor for DFS and DMFS. In addition, survivin combined with CD44 might be a candidate biomarker for the prediction of recurrence and survival in these patients.

Early studies on survivin expression were generally performed in patients undergoing surgery alone without preoperative treatment. Kawasaki et al. observed that survivin expression was strongly associated with bcl-2 expression and inversely correlated with apoptotic index (20). Survivin became an important predictive parameter of worse outcome, and several studies investigated this small novel apoptosis inhibitor. Almost all studies demonstrated significantly worse survival outcomes of patients with survivin-overexpressing tumors (21), except for one study (22). Recently, the need to find biomarkers that can predict radiosensitivity and treatment response before treatment has increased, since preoperative CRT followed by surgery has become the standard treatment for locally advanced rectal cancer (2). Several studies investigated survivin expression in pretreatment biopsy specimens and demonstrated there was no association between survivin expression and treatment response to preoperative CRT or prognosis $(23,24)$. In contrast, Kim et al. reported that survivin overexpression was significantly associated with poor down-staging (25), and Takasu et al. also recognized significant inverse correlation between preoperative CRT response and survivin expression (26). In the present study, a significant association was found between survivin overexpression and TRG. In addition, survivin overexpression was related to poor prognosis.

CSCs were shown to be involved in tumor progression, relapse and treatment resistance $(13,27-28)$. EMT has recently been considered to be associated with aggressive phenotype and metastatic potential of cancer cells $(12,29$ 30). We investigated immunohistochemistry of CD44, CD133, E-cadherin, TWIST1, and the association of their 

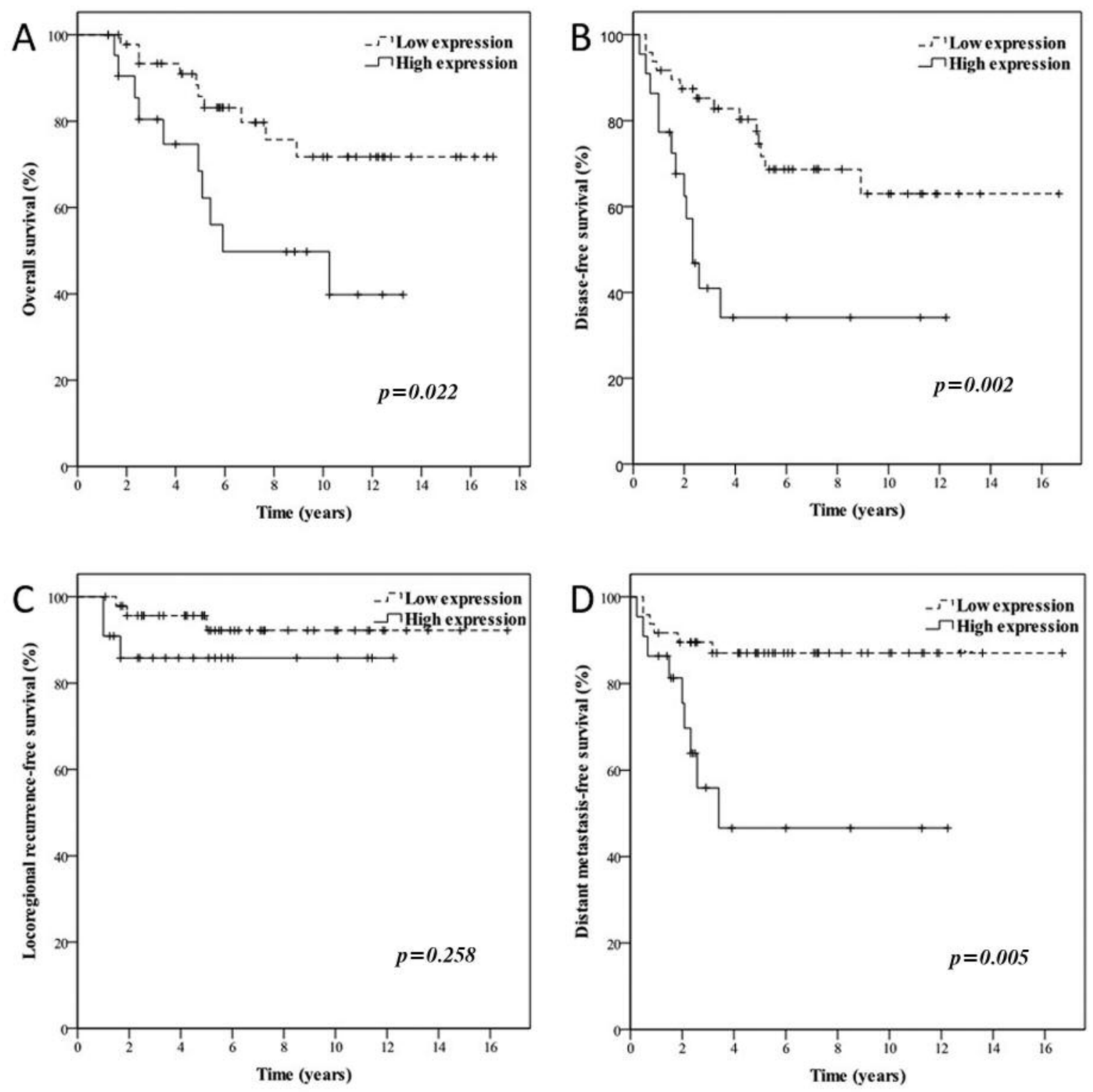

Figure 2. Kaplan-Meier curves for overall (A), disease-free (B), locoregional recurrence-free $(C)$, and distant metastasis-free $(D)$ survival according to survivin expression.

expression with treatment response and survival outcomes was evaluated. Expression of these four single molecular markers was not associated with TRG, down-staging, and survival outcomes, despite evidence supporting that the CSC/EMT phenotype is related to poor treatment response and poor prognosis in patients with rectal cancer $(19,31)$. However, discrepancies can exist between studies, and these discrepancies may be associated with the characteristics of antibody used (monoclonal vs. polyclonal), methodology, scoring criteria, treatment method, sample size, and followup period.
Accumulating evidence supports the notion that survivin overexpression is associated with CSC/EMT phenotypes $(19,21,32-33)$. Several mechanisms such as DNA damage repair capability, longer $G_{2}$ arrest, reactive oxygen species mechanism as well as apoptosis, are associated with the therapeutic resistance of CSCs (34). Therefore, studies have investigated the relationship between survivin, which plays an important role in suppressing caspase-mediated apoptosis, and the CSC phenotype. Kim et al. reported that interleukin-4 was significantly up-regulated in CD133positive cells compared to CD133-negative cells, and 

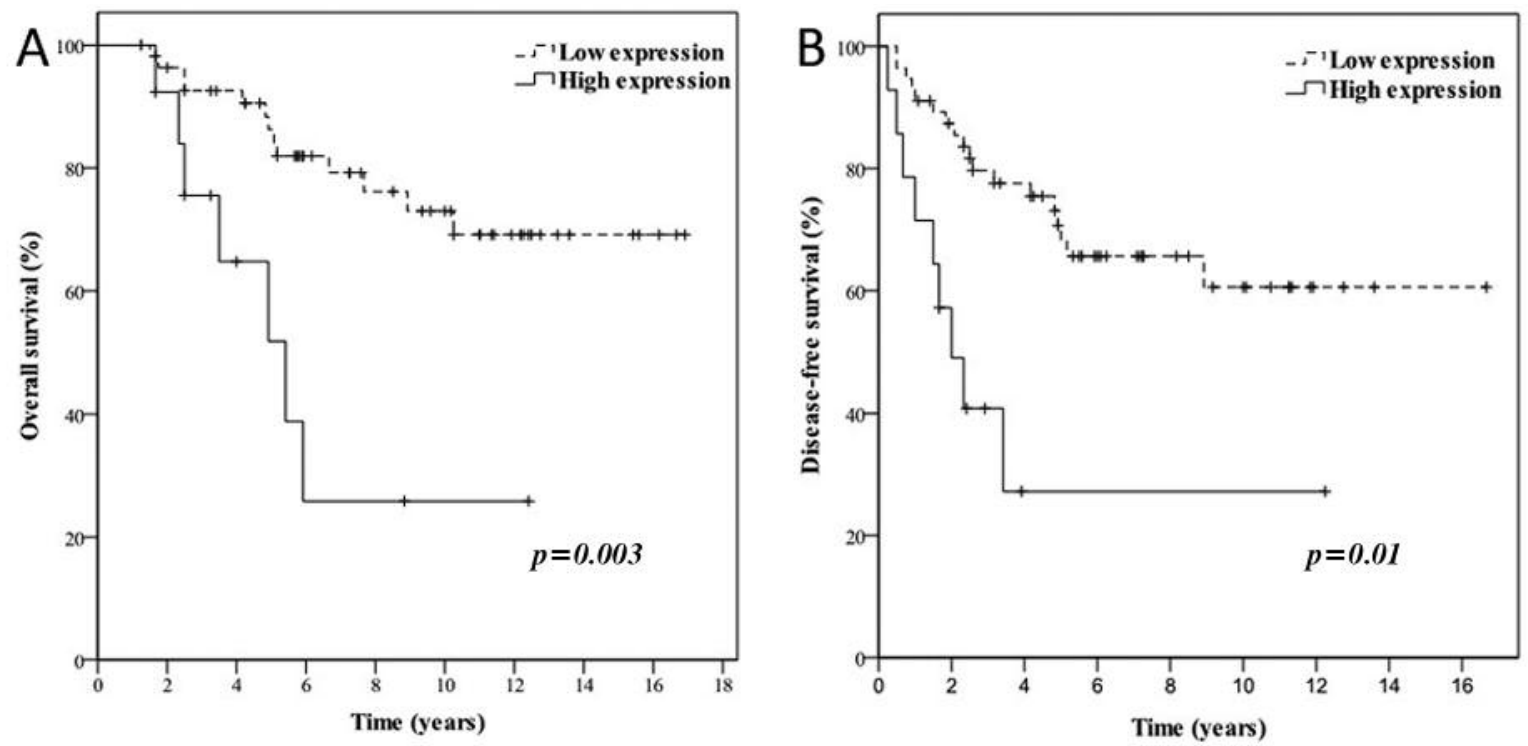

Figure 3. Kaplan-Meier survival curves for overall (A) and disease-free (B) survival according to combined survivin/CD44 expression.

Table IV. Univariate analysis for overall (OS), disease-free (DFS), and distant metastasis-free (DMFS) survival according to markers and combined markers expression

\begin{tabular}{|c|c|c|c|c|c|c|c|c|c|}
\hline \multirow[b]{2}{*}{ Variable } & \multicolumn{3}{|c|}{ OS } & \multicolumn{3}{|c|}{ DFS } & \multicolumn{3}{|c|}{ DMFS } \\
\hline & HR & $95 \% \mathrm{CI}$ & $p$-Value & HR & $95 \% \mathrm{CI}$ & $p$-Value & HR & $95 \% \mathrm{CI}$ & $p$-Value \\
\hline Survivin & 2.79 & $1.16-6.72$ & 0.022 & 3.35 & $1.55-7.25$ & 0.002 & 4.50 & $1.58-12.83$ & 0.005 \\
\hline CD44 & 1.70 & $0.65-4.45$ & 0.279 & 1.86 & $0.78-4.39$ & 0.159 & 1.23 & $0.42-3.60$ & 0.705 \\
\hline CD133 & 1.30 & $0.53-3.18$ & 0.569 & 1.31 & $0.61-2.81$ & 0.481 & 1.16 & $0.42-3.20$ & 0.775 \\
\hline E-cadherin loss & 0.59 & $0.20-1.77$ & 0.346 & 0.84 & $0.32-2.22$ & 0.721 & 0.76 & $0.21-2.70$ & 0.673 \\
\hline Twist1 & 1.52 & $0.60-3.84$ & 0.374 & 1.33 & $0.62-2.87$ & 0.468 & 1.33 & $0.47-3.74$ & 0.589 \\
\hline Survivin+/CD44+ & 4.25 & $1.66-10.89$ & 0.003 & 3.89 & $1.69-8.94$ & 0.001 & 3.89 & $1.36-11.10$ & 0.011 \\
\hline Survivin+/CD133+ & 2.66 & $1.09-6.51$ & 0.032 & 3.56 & $1.59-7.95$ & 0.002 & 4.20 & $1.50-11.73$ & 0.006 \\
\hline Survivin+/E-cadherin loss & 2.37 & $0.98-5.72$ & 0.055 & 3.20 & $1.47-6.93$ & 0.003 & 5.18 & $1.82-14.78$ & 0.002 \\
\hline Survivin+/TWIST1+ & 2.48 & $0.99-6.22$ & 0.054 & 3.85 & $1.71-8.69$ & 0.001 & 3.26 & $1.14-9.29$ & 0.027 \\
\hline Survivin+/CD44+/CD133+ & 4.33 & $1.52-12.29$ & 0.006 & 4.52 & $1.73-11.78$ & 0.002 & 3.84 & $1.21-12.20$ & 0.022 \\
\hline Survivin+/CD44+/TWIST1+ & 4.93 & $1.72-14.12$ & 0.003 & 5.05 & $2.01-12.68$ & 0.001 & 3.37 & $1.06-10.73$ & 0.040 \\
\hline Survivin+/CD44+/E-cadherin loss & 4.49 & $1.76-11.46$ & 0.002 & 4.51 & $1.96-10.39$ & $<0.001$ & 4.59 & $1.59-13.20$ & 0.005 \\
\hline Survivin+/CD133+/TWIST1+ & 2.41 & $0.93-6.29$ & 0.071 & 3.85 & $1.62-9.12$ & 0.002 & 3.31 & $1.12-9.82$ & 0.031 \\
\hline Survivin+/CD133+/E-cadherin loss & 2.25 & $0.90-5.64$ & 0.084 & 3.50 & $1.54-7.95$ & 0.003 & 5.07 & $1.81-14.2$ & 0.002 \\
\hline Survivin+/Twist $1+/$ E-cadherin loss & 2.07 & $0.79-5.39$ & 0.136 & 3.82 & $1.67-8.74$ & 0.002 & 3.95 & $1.38-11.28$ & 0.010 \\
\hline Survivin+/CD44+/CD133+/E-cadherin loss & 5.44 & $1.91-15.51$ & 0.002 & 6.60 & $2.59-16.83$ & $<0.001$ & 4.27 & $1.32-13.79$ & 0.015 \\
\hline Survivin+/CD44+/CD133+/TWIST1+ & 4.03 & $1.31-12.39$ & 0.015 & 4.06 & $1.47-11.16$ & 0.007 & 3.01 & $0.84-10.73$ & 0.090 \\
\hline Survivin+/CD44+/TWIST1+/E-cadherin loss & 5.44 & $1.91-15.51$ & 0.002 & 6.60 & $2.59-16.83$ & $<0.001$ & 4.27 & $1.32-13.79$ & 0.015 \\
\hline Survivin+/CD133+/TWIST1+/E-cadherin loss & 1.99 & $0.72-5.48$ & 0.184 & 3.93 & $1.61-9.61$ & 0.003 & 4.22 & $1.42-12.57$ & 0.010 \\
\hline Survivin+/CD44+/CD133+/TWIST1+/E-cadherin loss & 4.52 & $1.48-13.84$ & 0.008 & 5.62 & $2.02-15.64$ & 0.001 & 4.03 & $1.12-14.52$ & 0.033 \\
\hline
\end{tabular}

HR, Hazard ratio; CI, confidence interval. Bold values indicate significant difference.

several studies showed that survivin was significantly associated with CSC phenotype in colorectal cancer tissue $(21,32)$. Moreover, recent evidence showed the association between apoptosis and EMT. Zhu et al. showed that
TWIST1 had an anti-apoptotic function as well as effects on EMT (19), and Vesuna et al. reported that an increased expression of TWIST1 inhibited apoptosis by suppressing proapoptotic genes (33). 
Table V. Multivariate analysis for overall survivaI according to positivity for survivin and CD44 expression.

\begin{tabular}{|c|c|c|c|c|}
\hline \multirow{2}{*}{$\begin{array}{l}\text { Variable } \\
\text { Survivin+/CD44+ }\end{array}$} & \multirow[t]{2}{*}{$\mathrm{HR}$} & \multicolumn{2}{|c|}{$95 \% \mathrm{CI}$} & \multirow[t]{2}{*}{$p$-Value } \\
\hline & & & & \\
\hline High vs. low & 6.45 & 1.87 & 22.25 & 0.003 \\
\hline \multicolumn{5}{|l|}{ Age } \\
\hline$>50 v s . \leq 50$ Years & 3.37 & 0.59 & 19.23 & 0.172 \\
\hline \multicolumn{5}{|l|}{ CEA } \\
\hline$>5 \mathrm{ng} / \mathrm{ml} v s . \leq 5 \mathrm{ng} / \mathrm{ml}$ & 2.91 & 0.88 & 9.70 & 0.081 \\
\hline \multicolumn{5}{|l|}{ Pathological T category } \\
\hline $3-4$ vs. $0-2$ & 0.34 & 0.05 & 2.16 & 0.255 \\
\hline \multicolumn{5}{|l|}{ Differentiation } \\
\hline $\mathrm{PD} v s . \mathrm{WD} / \mathrm{MD}$ & 1.65 & 0.38 & 7.16 & 0.502 \\
\hline \multicolumn{5}{|l|}{ Perineural invasion } \\
\hline Present $v s$. absent & 0.77 & 0.18 & 3.30 & 0.726 \\
\hline \multicolumn{5}{|c|}{ Circumferential resection margin } \\
\hline Positive $v s$. negative & 118.64 & 7.95 & 1769.21 & 0.001 \\
\hline \multicolumn{5}{|l|}{ TRG } \\
\hline $2-4$ vs. 0-1 & 0.17 & 0.04 & 0.77 & 0.022 \\
\hline \multicolumn{5}{|l|}{ T Down-staging } \\
\hline Yes vs. no & 0.47 & 0.10 & 2.27 & 0.349 \\
\hline
\end{tabular}

TRG, Tumor regression grade; HR, hazard ratio; CI, confidence interval; CEA, carcinoembryonic antigen; PD, poorly differentiated; WD, well-differentiated; MD, moderately differentiated. Bold values indicate significant difference.

Co-expression of biomarkers may be better at reflecting the degree of acquisition of biological behaviors of cancer cells $(5,14-15)$. In order to identify useful biomarkers predicting treatment response and prognosis, combined biomarker analysis of the altered expressions of five markers (survivin, CD44, CD133, E-cadherin, and TWIST1) was performed herein. The majority of significantly predictive biomarker combinations included co-expression of both survivin and CD44. Given this observation, we performed further evaluation of the survivin/CD44-positive phenotype to clarify whether this combined biomarker could predict tumor response. Tumors harboring positivity for both survivin and CD44 had poor response to preoperative CRT, that is, TRG $0-1 \quad(p=0.019)$. Five-year OS and DFS of patients with survivin/CD44-positive phenotype were 51.8\% and $27.2 \%$, respectively. However, for the remaining phenotypes corresponding values were $86.2 \%$ and $68.1 \%$, respectively. Multivariate analysis for OS revealed the group with survivin/CD44-positive phenotype had a 6.45 -fold increased risk of death $(p=0.003)$. The simultaneous expression of these biomarkers may reflect more aggressive biological behavior of rectal cancer than the alteration of single biomarkers.

Certain limitations exist to this study. Firstly, there are inherent limitations of retrospective studies such as a small number of patients and potential bias from physicians regarding treatment selection. However, there was no significant difference in the treatment regimens between the two groups. Secondly, in the present study, only nuclear expression of survivin was semi-quantitatively assessed. Qi et al. suggested that subcellular localization of survivin may have different protein functions and reported nuclear survivin expression was associated with a favorable outcome (35). It remains controversial whether the subcellular location of survivin is associated with prognosis and further evaluation is needed.

In conclusion, survivin overexpression in pretreatment biopsy specimens was an independent adverse prognostic factor for DFS and DMFS, and the survivin/CD44-positive phenotype was an independent predictor for a shorter OS in patients receiving preoperative CRT for rectal cancer. Further research with a large population is needed to validate these results.

\section{References}

1 Krook JE, Moertel CG, Gunderson LL, Wieand HS, Collins RT, Beart RW, Kubista TP, Poon MA, Meyers WC and Mailliard JA: Effective surgical adjuvant therapy for high-risk rectal carcinoma. N Engl J Med 324: 709-715, 1991.

2 Sauer R, Becker H, Hohenberger W, Rödel C, Wittekind C, Fietkau R, Martus P, Tschmelitsch J, Hager E and Hess CF: Preoperative versus postoperative chemoradiotherapy for rectal cancer. N Engl J Med 351: 1731-1740, 2004.

3 Sauer R, Liersch T, Merkel S, Fietkau R, Hohenberger W, Hess C, Becker H, Raab HR, Villanueva MT, Witzigmann H, Wittekind C, Beissbarth T and Rodel C: Preoperative versus postoperative chemoradiotherapy for locally advanced rectal cancer: Results of the German CAO/ARO/AIO-94 randomized phase III trial after a median follow-up of 11 years. J Clin oncol 30: 1926-1933, 2012.

4 Song JH, Jeong JU, Lee JH, Kim SH, Cho HM, Um JW, Jang HS, Korean Clinical Practice Guideline for Colon and Rectal Cancer Committee: Preoperative chemoradiotherapy versus postoperative chemoradiotherapy for stage II-III resectable rectal cancer: A meta-analysis of randomized controlled trials. Radiat Oncol J 35: 198-207, 2017.

5 Hanahan D and Weinberg RA: Hallmarks of cancer: The next generation. Cell 144: 646-674, 2011.

6 Lim SH, Chua W, Henderson C, Ng W, Shin JS, Chantrill L, Asghari R, Lee CS, Spring KJ, de Souza P: Predictive and prognostic biomarkers for neoadjuvant chemoradiotherapy in locally advanced rectal cancer. Crit Rev Oncol Hematol 96: 6780, 2015.

7 Loftas P, Arbman G, Sun X-F, Edler D, Syk E and Hallbook O: FXYD-3 expression in relation to local recurrence of rectal cancer. Radiat Oncol J 34: 52-58, 2016.

8 Kim TG, Park W, Choi DH, Park HC, Kim S-H, Cho YB, Yun SH, Kim HC, Lee WY and Lee J: Effect of leukocyte alteration on treatment outcomes following preoperative chemoradiotherapy in patients with rectal cancer. Radiat Oncol J 35: 217-226, 2017.

9 Shin S, Sung BJ, Cho YS, Kim HJ, Ha NC, Hwang JI, Chung $\mathrm{CW}$, Jung YK and Oh BH: An anti-apoptotic protein human survivin is a direct inhibitor of caspase- 3 and-7. Biochemistry 40: 1117-1123, 2001. 
10 Huang YJ, Qi WX, He AN, Sun YJ, Shen Z, Yao Y, Zhu Y, Li Y, Zhu S, Tang R, Liu Y and Li J: The prognostic value of survivin expression in patients with colorectal carcinoma: a meta-analysis. Jpn J Clin Oncol 43: 988-995, 2013.

11 Krieg A, Werner TA, Verde PE, Stoecklein NH and Knoefel WT: Prognostic and clinicopathological significance of survivin in colorectal cancer: A meta-analysis. PLoS One 8: e65338, 2013.

12 Kang Y and Massague J: Epithelial-mesenchymal transitions: Twist in development and metastasis. Cell 118: 277-279, 2004.

13 Butof R, Dubrovska A and Baumann M: Clinical perspectives of cancer stem cell research in radiation oncology. Radiother Oncol 108: 388-396, 2013.

14 Ryu HS, Park DJ, Kim HH, Kim WH and Lee HS: Combination of epithelial-mesenchymal transition and cancer stem cell-like phenotypes has independent prognostic value in gastric cancer. Hum Pathol 43: 520-528, 2012.

15 Oon ML, Thike AA, Tan SY and Tan PH: Cancer stem cell and epithelial-mesenchymal transition markers predict worse outcome in metaplastic carcinoma of the breast. Breast Cancer Res Treat 150: 31-41, 2015.

16 Edge SB, Byrd DR, Compton CC, Fritz AG, Greene FL and Trotti A (eds.): AJCC Cancer Staging Manual (7th ed). New York, NY: Springer, 2010.

17 Sinicrope FA, Ruan SB, Cleary KR, Stephens LC, Lee JJ and Levin B: BCL-2 and p53 oncoprotein expression during colorectal tumorigenesis. Cancer Res 55: 237-241, 1995.

18 Zhang N-H, Li J, Li Y, Zhang X-T, Liao W-T, Zhang J-Y, Li R and Luo R-C: Co-expression of CXCR4 and CD133 proteins is associated with poor prognosis in stage II-III colon cancer patients. Exp Ther Med 3: 973-982, 2012.

19 Zhu D-J, Chen X-W, Zhang W-J, Wang J-Z, Ouyang M-Z, Zhong Q, Liu C-C: TWIST1 is a potential prognostic marker for colorectal cancer and associated with chemoresistance. Am J Cancer Res 5: 2000-2011, 2015.

20 Kawasaki H, Altieri DC, Lu CD, Toyoda M, Tenjo T and Tanigawa N: Inhibition of apoptosis by survivin predicts shorter survival rates in colorectal cancer. Cancer Res 58: 5071-5074, 1998.

21 Kim ST, Sohn I, Do IG, Jang J, Kim SH, Jung IH, Park JO, Park YS, Talasaz A, Lee J and Kim HC: Transcriptome analysis of CD133-positive stem cells and prognostic value of survivin in colorectal cancer. Cancer Genomics Proteomics 11: 259-266, 2014.

22 Kalliakmanis JG, Kouvidou C, Latoufis C, Kouvatseas G, Anagnostakis D, Papatheodoridis G, Koskinas J and Archimandritis A: Survivin expression in colorectal carcinomas: Correlations with clinicopathological parameters and survival. Dig Dis Sci 55: 2958-2964, 2010.

23 Terzi C, Canda AE, Sagol O, Atila K, Sonmez D, Fuzun M, Gorken IB, Oztop I and Obuz F: Survivin, p53, and Ki-67 as predictors of histopathologic response in locally advanced rectal cancer treated with preoperative chemoradiotherapy. Int J Colorectal Dis 23: 37-45, 2008.
24 McDowell DT, Smith FM, Reynolds JV, Maher SG, Adida C, Crotty P, Gaffney EF, Hollywood D, Mehigan B, Stephens RB and Kennedy MJ: Increased spontaneous apoptosis, but not survivin expression, is associated with histomorphologic response to neoajuvant chemoradiation in rectal cancer. Int $\mathbf{J}$ Colorectal Dis 24: 1261-1269, 2009.

25 Kim K, Chie EK, Wu HG, Kim SG, Lee SH, Kang GH, Hyun $\mathrm{CL}$ and $\mathrm{Ha} \mathrm{SW}$ : High survivin expression as a predictor of poor response to preoperative chemoradiotherapy in locally advanced rectal cancer. Int J Colorectal Dis 26: 1019-1023, 2011.

26 Takasu C, Shimada M, Kurita N, Iwata T, Sato H, Nishioka M, Morimoto S, Yoshikawa K, Miyatani T, Kashihara H, Utsunomiya $\mathrm{T}$ and Uehara $\mathrm{H}$ : Survivin expression can predict the effect of chemoradiotherapy for advanced lower rectal cancer. Int J Clin Oncol 18: 869-876, 2013.

27 Nguyen LV, Vanner R, Dirks P and Eaves CJ: Cancer stem cells: an evolving concept. Nat Rev Cancer 12: 133-143, 2012.

28 Rich JN: Cancer stem cells in radiation resistance. Cancer Res 67: 8980-8984, 2007.

29 Thiery JP, Acloque H, Huang RY and Nieto MA: Epithelial-mesenchymal transitions in development and disease. Cell 139: 871-890, 2009.

30 Puisieux A, Valsesia-Wittmann S and Ansieau S: A TWIST for survival and cancer progression. Br J Cancer 94: 13-17, 2006.

31 Sprenger T, Conradi LC, Beissbarth T, Ermert H, Homayounfar K, Middel P, Rüschoff J, Wolff HA, Schüler P and Ghadimi BM: Enrichment of CD133-expressing cells in rectal cancers treated with preoperative radiochemotherapy is an independent marker for metastasis and survival. Cancer 119: 26-35, 2013.

$32 \mathrm{Li} \mathrm{W}$, Lee MR, Choi E and Cho MY: Clinicopathologic Significance of survivin expression in relation to CD133 expression in surgically resected stage II or III colorectal cancer. J Pathol Transl Med 51: 17-23, 2017.

33 Vesuna F, Lisok A, Kimble B and Raman V: Twist modulates breast cancer stem cells by transcriptional regulation of CD24 expression. Neoplasia 11: 1318-1328, 2009.

34 Rich JN: Cancer stem cells in radiation resistance. Cancer Res 67: 8980-8984, 2007.

35 Qi G, Tuncel H, Aoki E, Tanaka S, Oka S, Kaneko I, Okamoto M, Tatsuka M, Nakai $S$ and Shimamoto F: Intracellular localization of survivin determines biological behavior in colorectal cancer. Oncol Rep 22: 557-562, 2009.
Received October 30, 2018

Revised November 14, 2018

Accepted November 16, 2018 